\title{
Possible Discovery Channel for New Charged Leptons at the LHC
}

\author{
V. E. Özcan ${ }^{a}$, S. Sultansoy ${ }^{b, c}$ and G. Ünel ${ }^{d}$
}

May 30, 2018

\author{
${ }^{a}$ Department of Physics and Astronomy, University College London, London, UK. \\ ${ }^{b}$ Institute of Physics, Academy of Sciences, Baku, Azerbaijan. \\ ${ }^{c}$ TOBB ETU, Physics Department, Ankara, Turkey. \\ ${ }^{d}$ University of California at Irvine, Physics and Astronomy Department, Irvine, USA.
}

\begin{abstract}
We propose a channel for the possible discovery of new charged leptons at the Large Hadron Collider. The proposed final state contains three same-sign leptons, making this new channel practically backgroundless. The method is illustrated for two different cases: the four-family Standard Model and the Grand Unified Theory based on the E6 gauge group. An example study taking $250 \mathrm{GeV}$ as the charged lepton mass shows that in both models, about 8 signal events can be expected at $14 \mathrm{TeV}$ center-of-mass energy with $1 \mathrm{fb}^{-1}$ of integrated luminosity. Although the event yield might not be sufficient for detailed measurements of the charged lepton properties, it would be sufficient to claim discovery through a counting experiment.
\end{abstract}

\section{Introduction}

In search of what comes after the Standard Model (SM), a number of theories based on different ideas such as Supersymmetry, Extra-dimensions and Grand Unification have been proposed [1, 2, 3]. The upcoming experiments at the LHC will test the validity of such ideas by searching for the new interactions or the new particles (bosons or fermions) predicted by these models. Among the most studied beyond the SM theories predicting new fermions one can cite the 4-family Standard Model (SM4), unified models based on E6 gauge group (E6GUT) and Little Higgs models [4, 5, 6].

The quark sector of the predicted fermions has received a lot of attention and the discovery potential at the LHC, large due to copious QCD production, was extensively discussed [7, 8, 9]. The models like E6GUT and SM4 also predict new heavy leptons. Some early studies considered the pair production of the heavy leptons mediated by $\gamma / Z / Z^{\prime}$, as a means of discovery [10]. The smallness of the pair production cross-section, compared to the quark sector, and its dependency on the $Z^{\prime}$ mass required consideration of integrated luminosities corresponding to many years of LHC operation. It was also thought that the search for the new heavy leptons would benefit from the clean environment of future lepton colliders [11, 12]. However, lepton colliders of sufficiently high center-of-mass energy is unlikely to be operational before 2020. Therefore the discovery potential of the LHC has to be maximally exploited. Pursuing that goal, it was recently shown that the LHC could possibly discover the neutral lepton and determine its nature [13, 14]. In this paper, we propose a channel through which the new charged lepton can also be searched for at the LHC.

\section{Proposed Discovery Channel}

We approach the problem of discovering new heavy leptons under relatively model-independent assumptions. We will denote the generic charged heavy lepton with $L^{\mp}$ and the neutral one with $L^{0}$. The discovery channel we propose is $p p \rightarrow W^{\mp *} \rightarrow L^{\mp} L^{0}$, where the charged lepton is assumed to be heavier 


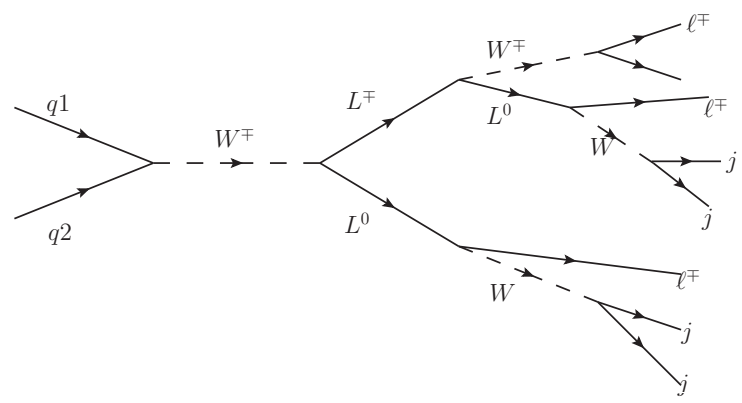

Figure 1: The proposed production and decay of new charged leptons at the LHC

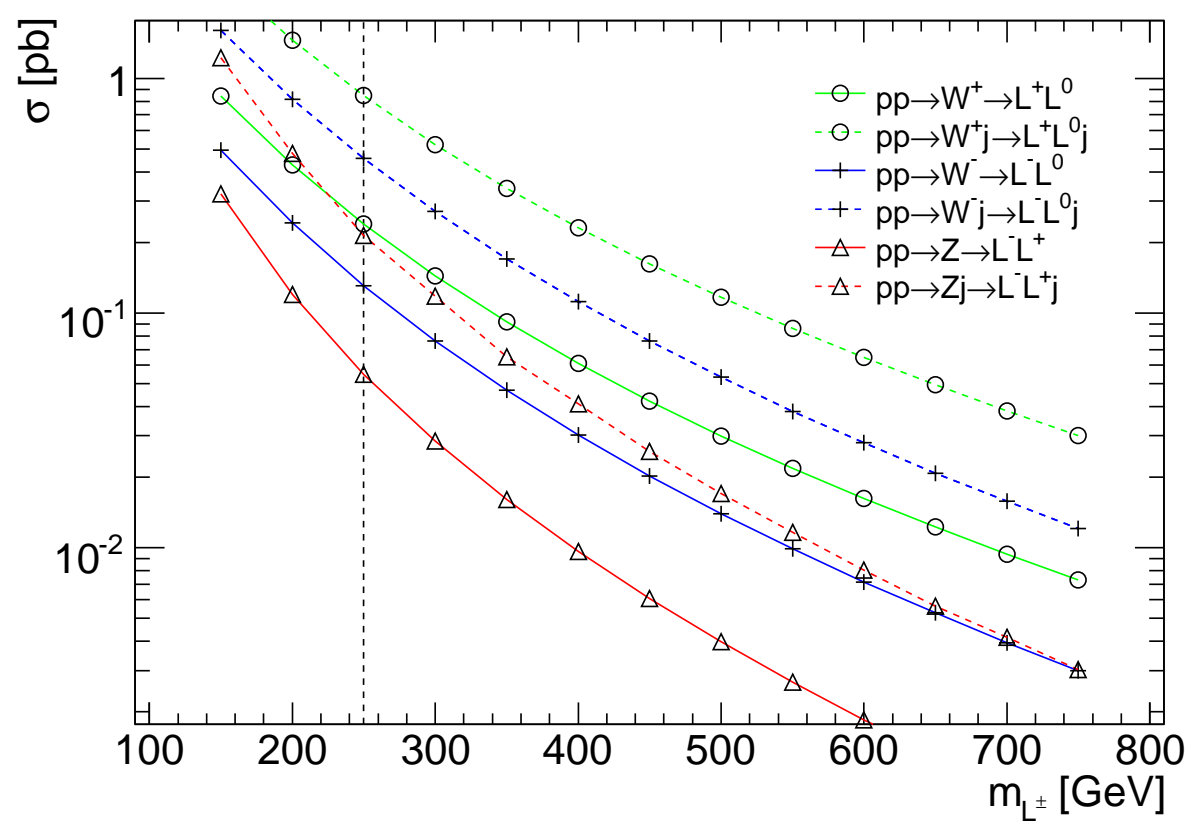

Figure 2: Tree-level cross-sections at the LHC running at $\sqrt{s}=14 \mathrm{TeV}$ for various production mechanisms of heavy leptons as functions of the charged lepton mass. The mass of the neutral heavy lepton, is taken to be $100 \mathrm{GeV}$. The vertical dashed line represents the reference scenario studied.

than the neutral one, $m_{L^{\mp}}>m_{L^{0}}$, resulting in the usual weak decay $L^{\mp} \rightarrow W^{\mp} L^{0}$, with the $W^{\mp}$ being real or virtual. In the best discovery scenario, the heavy neutral lepton is of Majorana type. In this case, the decays of the neutral leptons would yield two known leptons of same sign fifty-percent of the time: $L^{\mp} L^{0} \rightarrow W^{\mp} L^{0} L^{0} \rightarrow W^{\mp} W \ell^{\mp} W \ell^{\mp}$. When the primary $W^{\mp}$ boson, originating from $L^{\mp}$, decays leptonically ( $e$ or $\mu$ ), the resulting third lepton is also of the same sign as the first two, yielding in a final state with three same-sign leptons. This tree level production and decay process is illustrated in Fig. 1.

The cross-sections for the two possible $L^{\mp}$ production channels at the tree level have been calculated using the CompHEP 4.4.3 generator [26]. These channels are the pair production via $Z$ boson and the $L^{\mp} L^{0}$ associated production via the $W$ boson. The expected LHC cross-sections are shown in Fig. 2 as a function of the charged lepton mass. The mass of the heavy neutrino is taken as $100 \mathrm{GeV}$ in accordance with the recently published limits by the Particle Data Group [23]. It is seen that the Z-mediated channel has a lower cross-section and the consideration of the production process with an additional jet, shown by the dashed lines, increases the cross-sections and thus improves the discovery prospects. 


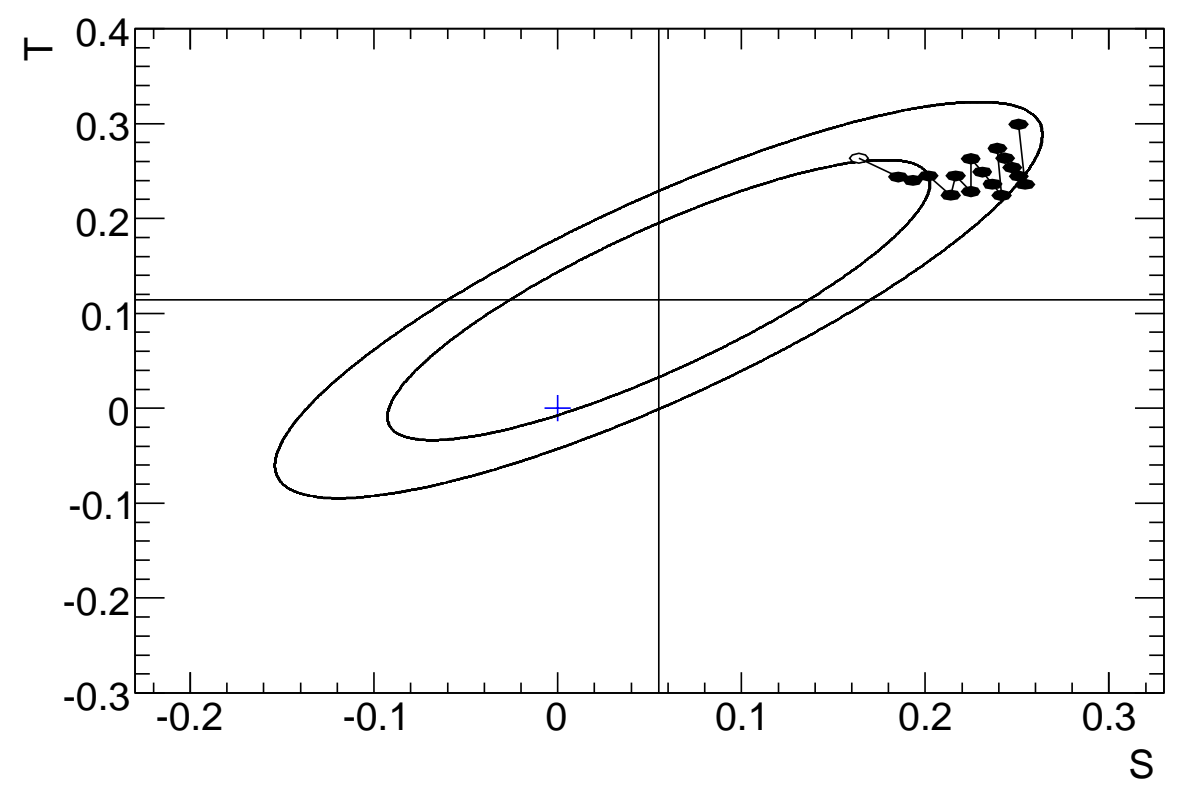

Figure 3: The 1 and 2 sigma error ellipses on the (S,T) parameters centered on the experimental value obtained by the LEP Electroweak Working Group. The SM, shown by the small cross, corresponds to $(\mathrm{S}=0, \mathrm{~T}=0)$ calculated with $m_{\text {top }}=170.9 \mathrm{GeV}$ and $m_{H}=115 \mathrm{GeV}$. The open circle is the $(\mathrm{S}, \mathrm{T})$ value calculated using Majorana neutrinos of 100 and $900 \mathrm{GeV}\left(\cos ^{2} \theta=0.9\right), m_{e_{4}}=250 \mathrm{GeV}, m_{u_{4}}=260 \mathrm{GeV}$, $m_{d_{4}}=260 \mathrm{GeV}$ and $m_{H}=115 \mathrm{GeV}$. The black circles represent the cases for increasing Higgs mass, from 150 to $900 \mathrm{GeV}$ in steps of $50 \mathrm{GeV}$ following the connecting line where the $u_{4}$ mass also slowly increases to $330 \mathrm{GeV}$.

\section{Application to 4th Generation Leptons}

The imminent operation of the LHC renewed the interest in a fourth Standard Model (SM) family [15]. The SM4 is predicted by the so called "flavor democracy" approach, which aims to explain the currently observed fermion mass hierarchy [16]. Furthermore, it gives an opportunity to explain the baryon asymmetry of the universe within the SM using the available CP violating phase in the extended CKM matrix [17]. The 3-family SM (SM3) leads to a ten orders of magnitude smaller CP violating phase compared to the observations [17]. The SM4 is obtained by extending the SM3 with an additional set of quarks and leptons denoted as: $u_{4}$ and $d_{4}$ for quarks, $e_{4}$ for the charged lepton and $v_{4}$ for the heavy neutrino. The fermionboson interaction vertices of the fourth family fermions are similar to the first three families. Although the masses and the mixings of the new fermions are not fixed by the SM, they are bound by the experimental and precision fit results [18, 19, 20, 23, 24].

The contributions to electroweak oblique parameters $\mathrm{S}$ and $\mathrm{T}$ arising from new fermions and the Higgs boson are calculated using the exact one-loop calculations given in References [20, 21, 22]. As a reference scenario, we consider Majorana-type heavy neutrinos of mass 100 and $900 \mathrm{GeV}$, yielding a mixing angle of $\cos ^{2} \theta=0.9$, and an equivalent Dirac mass of $v_{D}=300 \mathrm{GeV}$ in the nomenclature of Ref. [22]. The charged lepton mass is taken to be $250 \mathrm{GeV}$. For different values of the Higgs mass, a scan of the $u_{4}$ and $d_{4}$ mass values is performed to find the set yielding the $S, T$ values closest to the experimental value obtained by the LEP Electroweak Working Group. The results are shown in Fig. 3, where the ellipses represent the $68 \%$ and $95 \%$ confidence level constraints on the $\mathrm{S}$ and $\mathrm{T}$ parameters. We observe that for all Higgs masses between 115 and $900 \mathrm{GeV}$, the SM4 with Majorana neutrinos can provide solutions within the 2 sigma error ellipse. It is interesting to note that, for each Higgs mass value, the minimization process yields masses for the fourth family fermions that are within about $70 \mathrm{GeV}$ of each other as predicted by the DMM model [4]. For example for a Higgs of $300 \mathrm{GeV}$, the masses of the SM4 quarks are found to be 290 and $260 \mathrm{GeV}$ for $u_{4}$ and $d_{4}$, respectively. 


\subsection{Expectations for $e_{4}, v_{4}$}

In this section, we use a parameterization of the $4 \times 4$ PMNS matrix in agreement with the current neutrino oscillation data. In Ref. [25], the four-dimensional CKM matrix has been parameterized as a modification of $4 \times 4$ unit matrix, and the values for the three degrees of freedom in this parameterization have been extracted from the available experimental data on the fermion masses. The parameterization is common between the quark and lepton sectors and predicts $\operatorname{Br}\left(e_{4} \rightarrow W v_{4}\right) \simeq 1, \operatorname{Br}\left(v_{4} \rightarrow W \mu\right)=0.68$ for different values of the assumed unified Yukawa coupling coefficient and the corresponding values of the aforementioned parameters.

$p p \rightarrow W v_{4} v_{4} \rightarrow W W \mu W \mu$ has been studied as the particular final state where the considered decays are leptonic for the first and hadronic for the remaining two $W$ bosons. As mentioned previously, the two muons in the final state will be of the same sign fifty-percent of the time due to Majorana nature of the $v_{4}$. The branching fraction of these three same-sign lepton final states is $0.68^{2} \times 0.22 \times 0.68^{2} \times 0.5^{2} \simeq 1.2 \%$. The effective cross-section for this particular state is $4.44 \mathrm{fb}$ for $m_{e_{4}}=250 \mathrm{GeV}$ when no additional jets are taken into account. The same final state with an additional jet would enhance the cross-section by roughly a factor of four, yielding about 18 "backgroundless" events per $\mathrm{fb}^{-1}$.

\section{Application to E6 Leptons}

In the GUT (Grand Unified Theory) models, the $\mathrm{SM}$ gauge group $\mathrm{SU}(3)_{C} \times \mathrm{SU}(2)_{L} \times \mathrm{U}(1)_{Y}$ is embedded into a larger symmetry group, which is recovered at a higher scale. Such models predict new gauge bosons (e.g. $W^{\prime}, Z^{\prime}$ ) which are sought at the current accelerators and new fermions which may also be at the reach of the LHC. Among the most frequently studied ones, the super-string inspired, E6GUT model can be cited. It has a 27-plet per family which contains, among other fields, two fermion fields, denoted as $N$, and $E^{\mp}$ that could have the quantum numbers of a heavy neutral and charged lepton, respectively. We assume that only the members of a single 27-plet would be light enough to be accessible at the LHC. This assumption is pessimistic since further accessible families would increase the production cross-section. Secondly, we also assume the in-family mixings to be stronger than the inter-family mixings, motivated by current experimental results, giving a quasi diagonal CKM matrix.

The interesting properties of this model are the existence of flavour changing neutral currents (FCNCs) and the right-handed weak currents with vertices proportional to the mixing angle $\sin \theta_{R}$ which are severely constrained from the current precision data. Another severely constrained quantity is the difference between the mixing angles of the left components of the heavy leptons to their ordinary counterparts, $\delta \equiv \theta_{v_{e}}^{L}-\theta_{e}^{L}$, which has the potential of modifying the V-A structure of the model. We will take both of these mixings to be vanishingly small for the rest of this note, suppressing $E^{\mp} \rightarrow W^{\mp} v$ and $E^{\mp} \rightarrow Z e^{\mp}$ decays. In this case, the remaining $E^{\mp}$ decay mode is via the charged current process $E^{\mp} \rightarrow W^{\mp} N$, provided $m_{E^{\mp}}>m_{N}$.

\subsection{Expectations for $E^{\mp}, N$}

After the simplifying assumptions discussed in the previous section, the $E N W$ vertex becomes identical to the SM4 case allowing us to use the same computations for the E production at the LHC. The Dirac or Majorana nature of the neutrinos defines the ratio of branching fractions in the neutral current decays to its charged current decays. For Dirac-type ordinary neutrinos, and all mixing angles taken equal, this ratio is given as $\operatorname{Br}\left(N \rightarrow Z v_{e}\right) / \operatorname{Br}(N \rightarrow W e)=1 / 4$ [27]. The discovery channel becomes $p p \rightarrow W^{\mp} \rightarrow E^{\mp} N \rightarrow$ $W^{\mp} N N \rightarrow v_{\ell} \ell^{\mp} W e^{\mp} W e^{\mp}$ where $\ell$ could be an electron or a muon and the remaining two $W$ bosons could be reconstructed using their hadronic decays. The total branching fraction is calculated as $0.22 \times 0.75^{2} \times$ $0.5^{2} \times 0.68^{2}=1.4 \%$ making the effective cross-section for this particular state $5.2 \mathrm{fb}$ for $m_{E}=250 \mathrm{GeV}$ without considering the additional jets. The consideration of a single additional jet increases the expected signal yield to about 21 events per fb.

\section{Detector Effects and Estimates of the Backgrounds}

In this section, we consider the detector acceptance for the signal leptons and provide an order-of-magnitude estimate for backgrounds that might arise due to detector effects. For these purposes, we refer to the expected performance of the ATLAS Detector [28] as an example. In order to exploit both the single and 


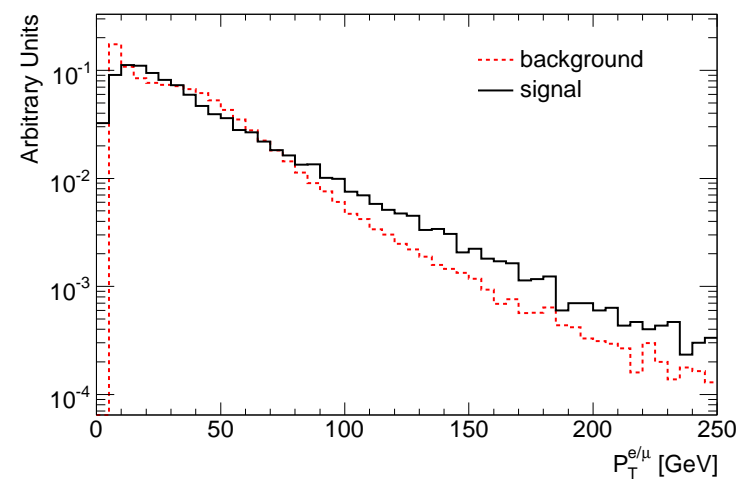

(a)

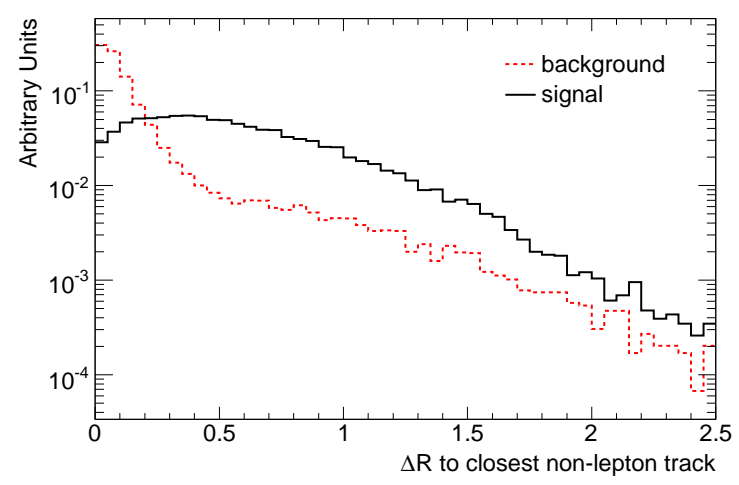

(c)

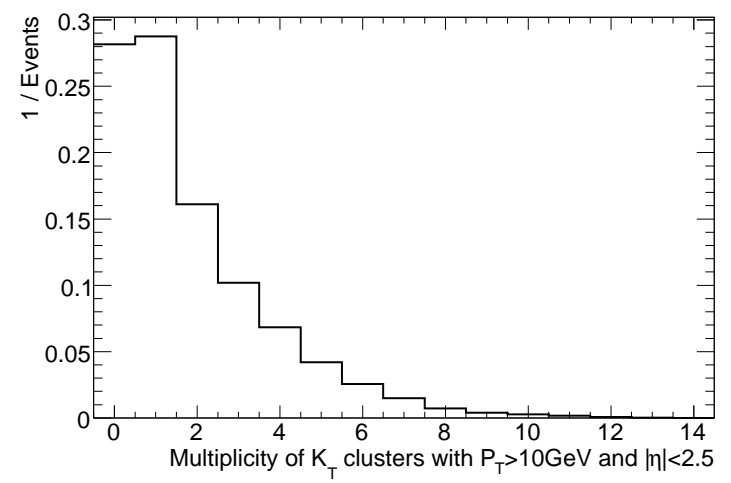

(b)

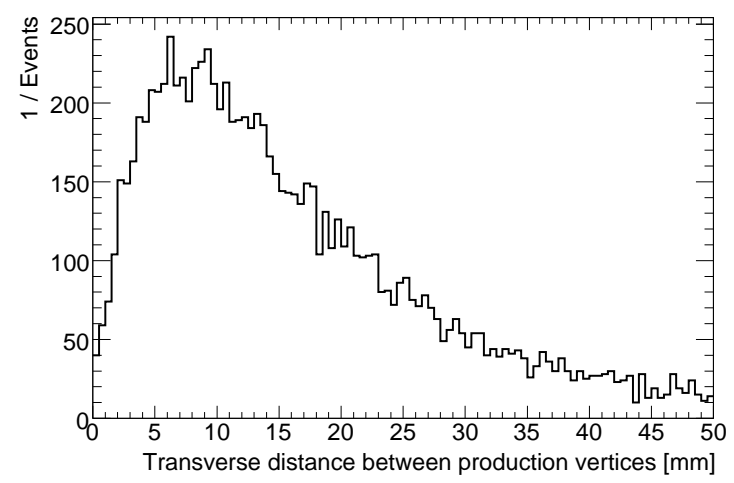

(d)

Figure 4: Distributions for variables relevant to the estimation of the backgrounds. (a) Transverse momenta for charged leptons from signal and $\ell^{+} \ell^{-} \ell^{+} v$ and $\ell^{+} \ell^{-} \ell^{-} \bar{v}$ background events. (b) Multiplicity for jets with $P_{T}>10 \mathrm{GeV}$ in $W+$ jet events. (c) $\Delta R$ to the closest non-lepton track for leptons in the signal events and for leptons originating from jets in $t \bar{t}$ events. (d) Maximum transverse distance between any of the two lepton production vertices in $t \bar{t}$ events.

di-lepton triggers for this detector, we require all leptons to have $P_{T}>10 \mathrm{GeV}$ and $|\eta|<2.5$, and at least one lepton to satisfy $P_{T}>20 \mathrm{GeV}$. Since the neutrinos of the reference scenario are relatively light, these requirements reject about half of the signal events. The requirements have similar or lower efficiencies for the three example background processes we discuss below, and, they are simply folded into our calculations.

First possible source of background is from SM3 processes to final states with three charged leptons of differing signs. We generate 75 thousand such events in the $\ell^{+} \ell^{-} \ell^{+} v$ and $\ell^{+} \ell^{-} \ell^{-} \bar{v}$ final states (where $\ell=$ $e, \mu$ and any combination of flavors is allowed) with Madgraph/MadEvent 4.2.0 [29]. Based on muon data in Ref. [28], charge mis-measurement rate is conservatively parameterized as $\varepsilon_{\text {mischarge }}=10^{-4+P_{T} / 200 \mathrm{GeV}}$, where $P_{T}$ is the lepton transverse momentum. Using this parametrization, a pseudo Monte Carlo study on the generated Madgraph events, for which the lepton $P_{T}$ are shown in Fig. 4a, yields the probability of obtaining three same-sign leptons to be $(2 \pm 0.5) \times 10^{-4}$. Multiplying by the total cross-section of $195.7 \pm 0.6 \mathrm{fb}$, we find that the expected number of background events is only $0.04 \mathrm{per} \mathrm{fb}^{-1}$.

The second possible source of background is from misidentification of jets as leptons. For an estimation of the size of this type of background, we refer to the expected performance of the maximum-likelihood based electron identification method, and take the jet rejection factor (inverse of the probability of misidentfiying jets as leptons) as $3.77 \times 10^{4}$. (The corresponding electron identification efficiency for this level of rejection is $77 \%$.) To convert this rejection factor into an estimate of the backgrounds, we generate $W+$ jet events with Pythia 6.4.14 [30] with multiple interactions on and the $W$ boson decaying to $e \bar{v}$ or $\mu \bar{v}$ with $P_{T}^{\text {lepton }}>10 \mathrm{GeV}$ and $\left|\eta^{\text {lepton }}\right|<2.5$. This particular type of events is interesting due to the high crosssection, $19.9 \pm 0.2 \mathrm{nb}$, and the presence of one true isolated lepton. On 10 thousand generated events, we 
run the $k_{T}$-jet algorithm [31] on hadron-level final-state particles and obtain the jet-multiplicity distribution shown in Fig. $4 \mathrm{~b}$ Using this distribution and the mentioned jet-rejection factor, we compute the probability of having two jets misidentified as leptons with the same sign as the true lepton in the event. Multiplying with the cross-section, the expected effective cross-section for this type of background is found to be $0.01 \mathrm{fb}$.

As the third example background source, we consider the $t \bar{t}$ production since the leptons from $b$ quark decays can the potentially emulate the signal. We generate 5 million $t \bar{t}$ events with Pythia 6.4.14. Lepton charge and jet misidentification probabilities, as described above, are also incorporated. For all lepton candidates, we apply an isolation requirement of $\Delta R>0.05$, where $\Delta R$ is the distance in the $\eta-\phi$ plane, between the lepton candidate and the closest non-lepton track of $P_{T}>1 \mathrm{GeV}$ (Fig. 4c). For the track isolation calculations the tracking efficiency is also folded in; defined conservatively as $\varepsilon_{\text {tracking }}=0.88-\frac{0.13 \times|\eta|}{2.5}$. The three-same-sign-lepton events in $t \bar{t}$ mostly originate from one $W$ boson decaying leptonically accompanied by at least 2 leptons from $b$-jets. To eliminate such cases, we also reject any event which has 2 or more leptons with $\Delta R<0.2$. In order to further reduce the background, the lepton vertex information can also be used: the signal leptons are produced at the interaction point, a feature not found in $t \bar{t}$ events. Therefore the 3 lepton candidates from $t \bar{t}$ events would fail to be fit into a single production vertex. Lacking the full detector simulation and proper vertexing algorithms, we resort to a simple selection based on the maximum transverse distance (MTD) between any two of the lepton production vertices. The expected secondary vertex transverse position resolution of the ATLAS Detector, as measured in $J / \Psi \rightarrow \mu^{+} \mu^{-}$events, is $0.17 \mathrm{~mm}$ or better. The MTD in $t \bar{t}$ events are significantly higher than this value, as shown in Fig. 4d, therefore requiring $\mathrm{MTD} \geq 0.4 \mathrm{~mm}$ (a distance corresponding to a value better than $2 \sigma$ ) would eliminate a large portion of the $t \bar{t}$ background without any significant loss in signal events. The acceptance, trigger and selection requirements have an overall efficiency of $40 \%$ on signal events, whereas only 1 out of 5 million $t \bar{t}$ events survives. Hence, using the predicted NLO cross section of $833 \mathrm{pb}$, we expect 0.17 events per fb ${ }^{-1}$ from $t \bar{t}$ background.

It can be argued that the selected processes are only partially representative of the full set of all sources of backgrounds. However, it is important to remember that for a full analysis on real data, it will be possible to reduce these and other backgrounds by optimizing the lepton identification criteria, by requiring one or more leptons to have relatively high momenta, by vetoing lepton pairs with invariant close to the nominal $Z$ mass, by using $b$-tagging, etc. These crude estimates highlight that the backgrounds are indeed expected to be an order of magnitude smaller than the predicted signals.

\section{Conclusion}

It is seen that the charged leptons of the fourth SM family and the E6GUT models can be searched using the $3 \ell+\geq 4 j+M E T$ final state where the leptons $(\ell)$ are of the same sign. The charged leptons are expected to be 3 same sign muons $50 \%$ of the time and 2 same sign muons +1 same sign electron for the other $50 \%$ for the first model. For the second model electrons and muons in the final state are to be interchanged.

Although the detector effects and the reconstruction efficiency have not been exactly calculated in this paper, the essentially backgroundless nature of this search channel, confirmed by the order magnitude estimates, makes it promising for the LHC, before the operation of the corresponding lepton colliders. For an inclusive study of our reference scenario, taking into account the selection criteria described in the previous section, the first (second) model yields 7 (8) events at $14 \mathrm{TeV}$ center of mass energy with $1 \mathrm{fb}^{-1}$ of integrated luminosity. Although the event yield might not be sufficient for detailed measurements of the charged lepton properties, it is sufficient to claim discovery through a counting experiment. For example, even if the SM backgrounds were an order of magnitude larger than our rudimentary estimate of 0.22 events/fb, the expected significance would still be over $3.5 \sigma$ using $\sqrt{2\left[(s+b) \ln \left(1+\frac{s}{b}\right)-s\right]}$ as the estimator. Moreover, the consideration of additional charged bosons $\left(W^{\prime}\right)$ as the mediator in the $s$ channel and of other decay modes of the secondary $W$ bosons can further increase the effective cross-section, reducing the integrated luminosity needed to discover the charged heavy leptons.

\section{Acknowledgments}

S.S. acknowledges the support from the Turkish State Planning Committee under the contract DPT2006K120470. G.Ü.'s work is supported in part by U.S. Department of Energy Grant DE FG0291ER40679. V.E.Ö. acknowledges financial support from the UK Science and Technology Facilities Council. 


\section{References}

[1] H. P. Nilles, Phys. Rept. 110, 1 (1984) and references therein.

[2] L. Randall, R. Sundrum, Phys. Rev. Lett. 83, 3370 (1999) and references therein.

[3] R. Blumenhagen, S. Moster and T. Weigand, Nucl. Phys. B 751, 186 (2006) [arXiv:hep-th/0603015].

[4] H. Fritzsch, Phys. Lett. B 289, 92 (1992); A. Datta, Pramana 40, L503 (1993); A. Celikel, A. Ciftci and S. Sultansoy, Phys. Lett. B 342, 257 (1995).

[5] F. Gursey, P. Ramond and P. Sikivie, Phys. Lett. B 60, 177 (1976); F. Gursey and M. Serdaroglu, Lett. Nuovo Cimento 21, 28 (1978).

[6] M. Schmaltz, Nucl. Phys. Proc. Suppl. B 117, 40 (2003).

[7] ATLAS Detector and Physics Performance Technical Design Report. CERN/LHCC/99-14/15 (1999), section 18.2; ; E. Arik et.al., Phys. Rev. D 58, 117701 (1998).

[8] B. Holdom, JHEP 0608, 076 (2006); B. Holdom, JHEP 0703, 063 (2007); B. Holdom, arXiv:0705.1736 (2007).

[9] V. E. Ozcan, S. Sultansoy and G. Unel, Eur. Phys. J. C 57, 621 (2008).

[10] C.Alexa and S.Dita, ATL-PHYS-2003-014 (2003).

[11] E. Accomando et al. [CLIC Physics Working Group], [arXiv:hep-ph/0412251].

[12] R. Ciftci, A. K. Ciftci, E. Recepoglu and S. Sultansoy, Turk. J. Phys. 27, 179 (2003) |arXiv:hep-ph/0203083].

[13] T. Cuhadar-Donszelmann, M. K. Unel, V. E. Ozcan, S. Sultansoy and G. Unel, JHEP 0810, 074 (2008) [arXiv:0806.4003 [hep-ph]].

[14] F. del Aguila and J. A. Aguilar-Saavedra, arXiv:0808.2468 [hep-ph]].

[15] B. Holdom, W. S. Hou, T. Hurth, M. L. Mangano, S. Sultansoy and G. Unel, [arXiv:0904.4698 [hep$\mathrm{ph}]$.

[16] S. Sultansoy, AIP Conf. Proc. 899, 49 (2007) [arXiv:hep-ph/0610279] and references therein.

[17] G. W. S. Hou, ICHEP 08 Conf. Proc., arXiv:0810.3396/hep-ph].

[18] G.D. Kribs, T. Plehn, M. Spannowsky and T.M.P. Tait, Phys. Rev. D 76, 075016, (2007) [arXiv:0706.3718].

[19] V. A. Novikov, L. B. Okun, A. N. Rozanov and M. I. Vysotsky, Phys. Lett. B 529, 111 (2002) |arXiv:hep-ph/0111028]; V. A. Novikov, L. B. Okun, A. N. Rozanov and M. I. Vysotsky, JETP Lett. 76, 127 (2002) [arXiv:hep-ph/0203132].

[20] H. J. He, N. Polonsky and S. F. Su, Phys. Rev. D 64, 053004 (2001) [arXiv:hep-ph/0102144].

[21] M. E. Peskin and T. Takeuchi, Phys. Rev. D 46, 381 (1992).

[22] B. A. Kniehl and H. G. Kohrs, Phys. Rev. D 48, 225 (1993).

[23] C. Amsler et al., Phys. Lett. B 667, 1 (2008).

[24] P.Q. Hung and Mark Sher, Phys. Rev. D 77, 037302 (2008) arXiv:0711.4353 [hep-ph].

[25] A. K. Ciftci, R. Ciftci and S. Sultansoy, Phys. Rev. D 72053006 (2005).

[26] A. Pukhov, arXiv:hep-ph/0412191 (2004); E. Boos et al. (CompHEP Collaboration), Nucl. Instrum. Meth. A 534, 250 (2004). 
[27] J. Hewett and T. Rizzo, Phys. Rep. 183, 193 (1989).

[28] ATLAS Collaboration, Expected Performance of the ATLAS Experiment, Detector, Trigger and Physics, CERN-OPEN-2008-020 (2009).

[29] J. Alwall et al., MadGraph/MadEvent v4: The New Web Generation, JHEP 0709 (2007) 028.

[30] T. Sjöstrand, S. Mrenna and P. Skands, PYTHIA 6.4 Physics and Manual, JHEP 0605 (2006) 026.

[31] S. Catani, Yu. L. Dokshitzer, M. H. Seymour and B. R. Webber, Nucl. Phys. B 406 (1993) 187. 